\title{
COMPARATIVE PERFORMANCE ANALYSIS OF MYSQL AND SQLITE RELATIONAL DATABASE MANAGEMENT SYSTEMS IN WINDOWS10 ENVIRONMENT
}

\author{
Jayesh Umre $^{1}$, Kunal Batra ${ }^{2}$, Vivek Vaidya ${ }^{3}$
}

\begin{abstract}
Different types of database systems are available with same or different functionality. Enormous amount of data flow in our surrounding makes Relational Database Management System the most important and popular tool to manage these data's. Open-source RDBMS systems like SQLite, are gaining popularity day by day. This paper is an attempt to set a benchmark in comparing the performance of MySQL against SQLite in Windows 10 Environment. This test was performed to find which open source RDBMS is best for the application like RGPV online quiz system in which multiple choice types of questions are used. MCQ type application required mainly the fetch of data from backend to the user screen. So main concentration of this paper was to find the database which is best suited for the multiple choice questions type application like RGPV online quiz system.
\end{abstract}

Keywords-MySQL, SQLite, Peformance Analysis, ROQS.

\section{INTRODUCTION}

ROQS is a RGPV Online quiz system designed for RGPV University students for performing the practice test of student's internal and external practical examinations. It stores information about the academia of the engineering students according to their University syllabus in the form of (MCQ) multiple choice questions, which include information about the question number, question, answer, wrong answer, their result etc. RGPV online quiz system designed in such a way that the students as well as the faculties feel comfortable in using the software. RGPV online quiz system enables students to register themselves online or in a network, as well as register through faculty manually. The intension of this project is to develop software which is more user friendly, and can be efficiently used by people in different roles.

We saw the development of this project as an opportunity for analyzing the comparative performance of MySQL and SQLite. The main focus of this paper is to analyze the performance of the system in two databases namely-MySQL and SQLite and to discover which database is well suited to work with this system.

\footnotetext{
${ }^{1}$ Department of Computer Science, TSEC Burhanpur, India

${ }^{2}$ Department of Computer Science, TSEC Burhanpur, India

${ }^{3}$ Department of Computer Sc. \& Engg., J.M.Govt.Polytechnic College Burhanpur, India
} 


\section{WHY MYSQL AND SQLITE}

MySQL and SQLite are two of the most popular RDBMS systems. MySQL is the most used database system in organizations, while MySQL is the third most popular [5]. In overall-use rankings however, MySQL is the second most popular database system after Oracle DB, while SQLite is the seven most popular [6]. The mismatch in these ranking is considered because MySQL is seen more in website implementation while SQLite is considered a tool that appeals most often to individuals interested in managing databases associated with their mobile applications[9].

MySQL and SQLite as the databases were selected based on the convenience of the developers, available resources and the fact that the project happens to use the relational model in the database.

\section{A. $M y S Q L$}

MySQL is an open-source RDBMS which ranked second in the world as second most used RDBMS in the world [6], and the most popular RDBMS system among all open-source RDBMS systems. Platform independency makes it great to be implementing in any application. The various features of MYSQL and SQLite are as given bellows-

Features of MySQL:

1) It provides cross-platform support.

2) MySQL execution is very fast.

3) MySQL supports many data types.

4) In MySQL memory allo0cation system is very fast.

5) MySQL supports both fixed length and variable length records.

\section{B. SQLite}

SQLite is an open-source embedded database, which is written in C language by D. Richard Hipp. SQLite is a lightweight database as compare to usual databases such as SQL Server, Oracle etc. SQLite is a software library that implements a self-contained, zero configuration, server less, transactional SQL database engine. SQLite is the most widely deployed SQL database engine in the world.

Features of SQLite [10][11][12]:

1) SQLite is an open source embedded database with self-contained library.

2) SQLite is zero configuration means no setup or administration needed.

3) SQLite is server less means it does not require a separate server process or system to operate.

4) SQLite can preserve any type of data means it required no data type.

5) SQLite doesn't rely on any operating system, its fast, efficient and scalable.

6) SQLite provides many supports to APIs.

\section{RELATED WORKS}

In [1], the performance comparison for data storage of Resort management system with two databases namely My SQL and SQL Server was done. The authors have made a detailed study about data storage in Resort management system and have made an analysis of data storage in both the databases. The authors have considered execution time as a parameter for performance analysis. 
In [2], performance comparison between Dbo4 and MySQL was done. They have done comparison of these two systems on the basis of execution time and memory consumption as a parameter for performance analysis.

In [3], performance comparison between MySQL and Virtuoso universal Server 6 triple stores was done. They have done comparison of these two systems on the basis of average read and write times.

In [4], performance comparison for row-storage vs column-storage in databases, using SQL Server and Oracle DB as test database system was done. This paper had analyzed the execution time where a sequence of five SELECT queries against database systems were executed, using MATLAB as a front-end.

\section{PROPOSED METHODOLOGY}

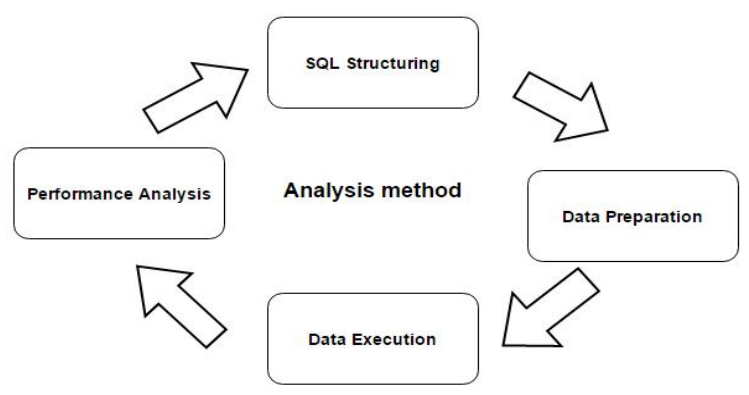

Fig. 1: The analysis method.

\section{A. SQL Structuring}

As mentioned before, the purpose of this paper is to find out the database system that works best for the RGPV online quiz system. RGPV online quiz system is accessed by many users at the same time so the SQL structure should be simple and syntax must be fast executing. So selecting the best SQL logic for the RGPV online system was the tough task to perform the student online practical examinations.

\section{B. Data Preparation}

RGPV online quiz system acts as a tool for data collection in this performance study. Daily interaction of the user with the system makes database to be executed regularly and error free. So the process of data cleaning needed to be removing corrupted and inappropriate data. Regular data checking needed to be performed. Data used in RGPV online system must be actual data as per the syllabus of RGPV University.

\section{Data Execution}

RGPV online quiz system concentrates on the performance analysis of the backend with respect to the databases MySQL and SQLite. The different modules of the system are executed in both the databases MySQL and SQLite. The execution time (time taken by the database to return the result of a query) is recorded in each case. This record is later utilized to find which database is best suitable for RGPV online quiz system with respect to the time it takes to execute the query.

\section{Performance Analysis}

The database is executed, using both the databases MySQL and SQLite and performance is analyzed based on the time it takes to complete its query execution. The query execution times are obtained and then tabulated in Microsoft Excel for further processing. The 
Comparative Performance Analysis Of Mysql And Sqlite Relational Database Management Systems In Windows10 Environment

performance analysis is done on both the databases MySQL and SQLite and the database well suited for RGPV online quiz system is found out.

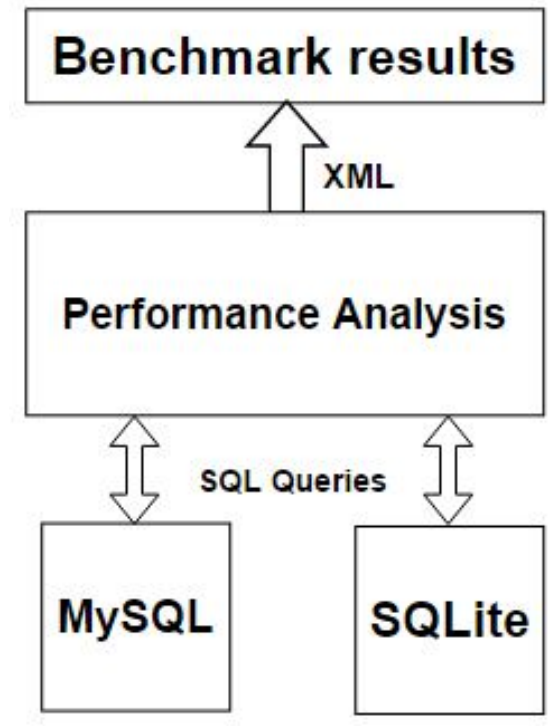

Fig. 2: design of the analysis system.

The "SQL Queries" [Fig. 2] in RGPV online quiz system is responsible for CRUD (Create, Read, Update and Delete) operations as well as the time taken to execute the opearations.

These entries provide us with data for the performance comparison between MySQL and SQLite.

\section{COMPARISON}

The Comparison test was performed on a Windows 10 64-bit machine running on an Intel® Core $^{\mathrm{TM}}$ i5-2430M CPU with a clock frequency of $2.4 \mathrm{GHz}$. And 4GB of RAM.

The MySQL 5.5 and SQLite Manager 3.5 were the respective versions of MySQL and SQLite to test the data. The data was first collected in SQLite, and then migrated to MySQL using the SQLite Manager export functionality.

Different SQL queries, namely SELECT, INSERT, UPDATE, and DELETE were executed on both and the execution time was recorded via the RGPV online quiz system.

The total numbers of rows we try to maintain more than 10000 to get performance result according to RGPV online quiz systems need.

The test cases were as follows -

\section{A. SELECT queries}

Same SELECT queries were executed on both MySQL and SQLite with same entries and same number of rows, with same name of table and same name of database. This Select query was executed 10 times with 10984 rows.

\section{B. INSERT queries}

On both MySQL and SQLite, the average time to insert 50 rows was calculated. In using INSERT query in both the databases MySQL and SQLite same data's was inserted with same number of queries and with same insertion order of data. 


\section{UPDATE queries}

On both MySQL and SQLite update query was executed 50 times on some rows with WHERE clause. The average of this 50 time query execution was found out to calculate the performance of MySQL and SQLite. Some UPDATE queries were run in between the middle rows and some UPDATE queries were run on the last number of rows.

\section{DELETE queries}

In case of DELETE queries as well, some rows were deleted one by one using WHERE clause within MySQL and SQLite database. And their execution time was recorded on the basis of which performance of both systems were analyzed. The DELETE queries were executed on rows randomly.

Delete query was executed 50 times on 10984 rows.

\section{RESULTS}

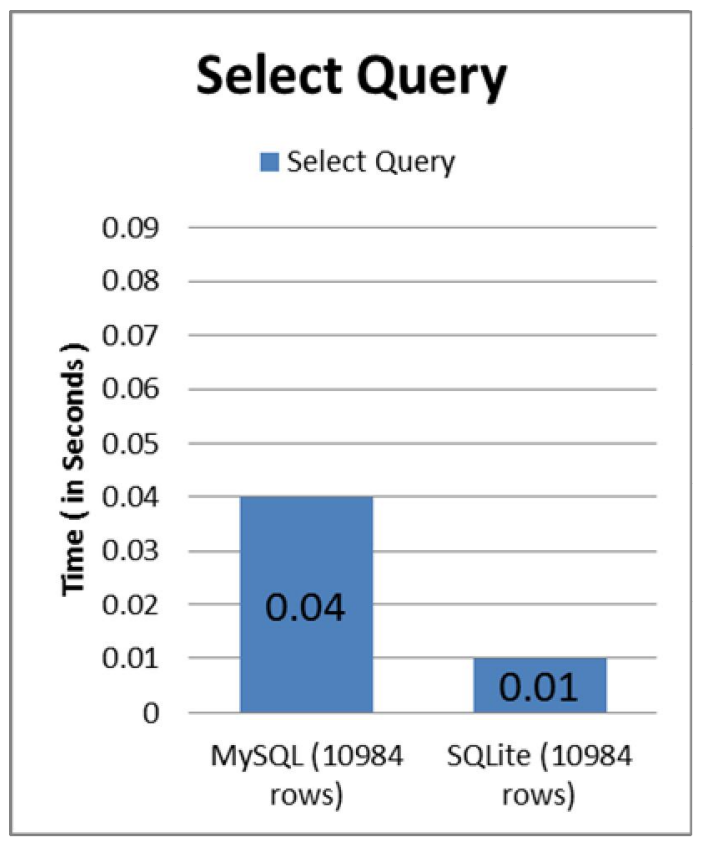

Fig. 3: Averages for a SELECT query. 
Comparative Performance Analysis Of Mysql And Sqlite Relational Database Management Systems In Windows10 Environment

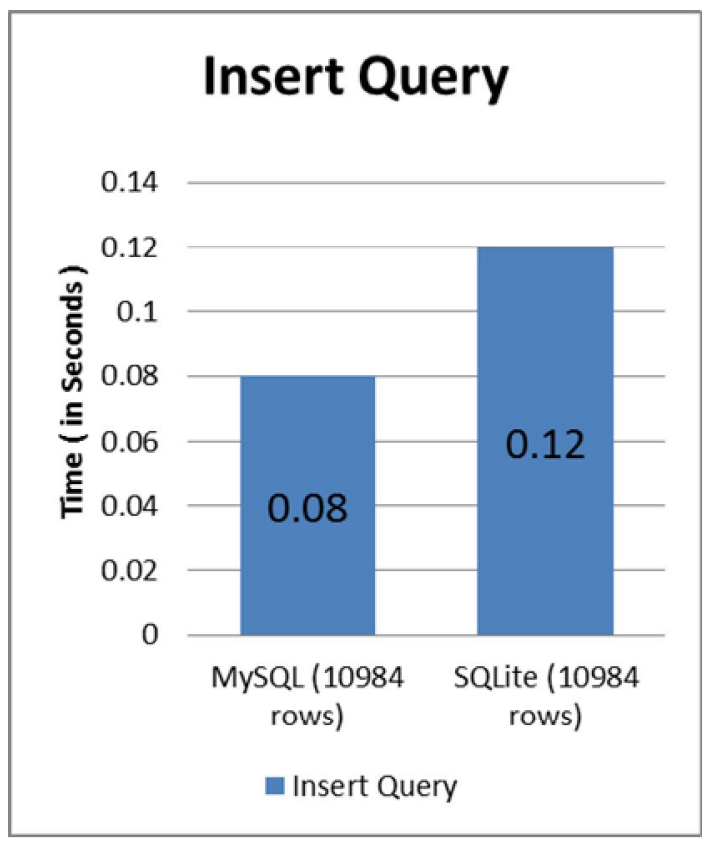

Fig. 4: Averages for INSERT query.

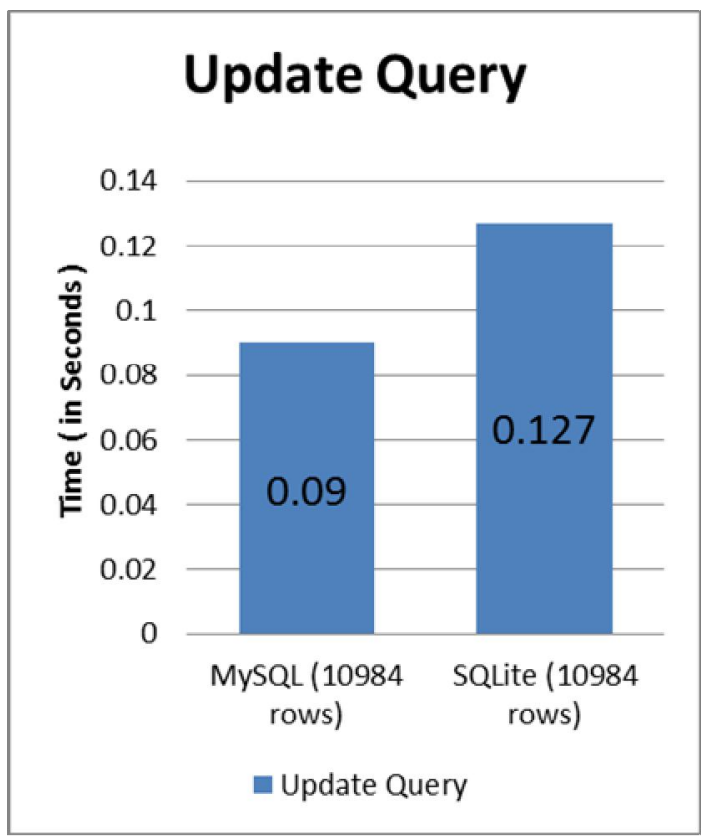

Fig. 5: Averages for UPDATE query. 


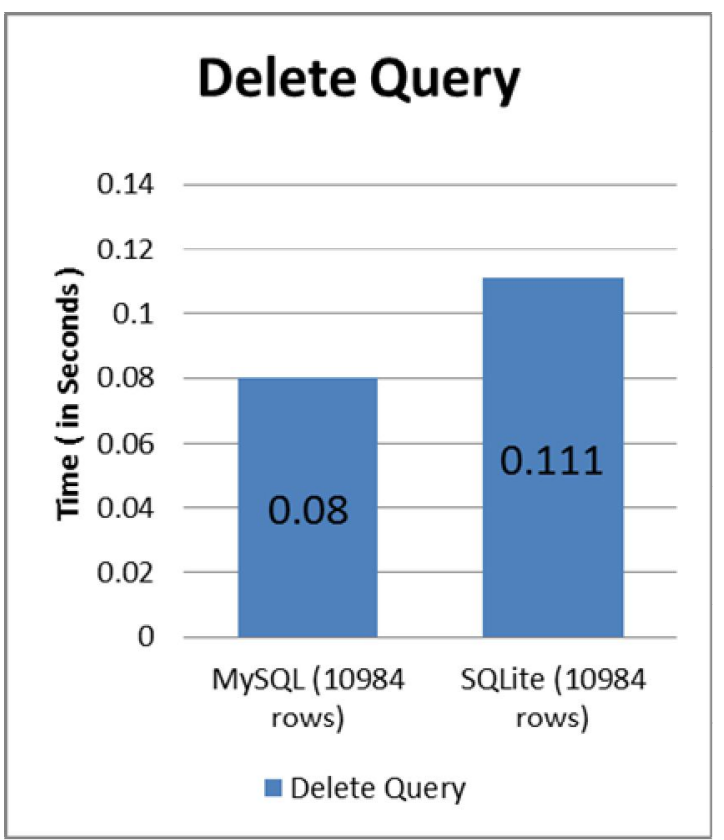

Fig. 6: Averages for DELETE query.

\section{CONCLUSION}

The purpose of this paper is to analyze the performance of two popular relational database management systems, MySQL and SQLite, in terms of time taken to respond to requests.

The results show that MySQL offers more performance than SQLite in terms of response time. In all the test cases, except SELECT queries [Fig 3], MySQL consistently took lesser time when compared to SQLite.

SQLite shows a bigger increase in time taken when the number of rows goes up. MySQL also allowed similar result, but the increase in time taken was not great as SQLite.

The most striking difference in performance was in SELECT query. In Fig 3, we can see that the time taken by MySQL is more than SQLite when dealing with 10984 rows.

In our future work, the increase in the scope of the analysis in terms of parameters, database systems, DBMS models, and execution environment will be considered. Also, the comparison of the execution time and memory consumption of RDBMS systems on both Windows and UNIX platform can be considered for further enhancement.

\section{References}

[1] A. Saikia, S. Joy, D. Dolma, R. Mary. R. "Comparative Performance Analysis of MySQL and SQL Server Relational Database Management Systems in Windows Environment”, Department of Computer Science, Christ University, Bengaluru, India. Noida, India, 2015.

[2] S. Kulshrestha and S. Sachdeva, "Performance Comparison for Data Storage - Db4o and MySQL Databases", Department of Computer Science and Engineering, Jaypee Institute of Information Technoloogy, Noida, India, 2014.. Kilintzis, N. Beredimas, and I. Chouvarda, "Evaluation of the performance of open-source RDBMS and triplestores for storing medical data over a web service".

[3] V. Kilintzis, N. Beredimas, and I. Chouvarda, "Evaluation of the performance of open-source RDBMS and triple stores for storing medical data over a web service".

[4] A.K. Dwivedi, C.S. Lamba, S. Shukla, "Performance Analysis of Column Oriented Database versus Row Oriented Database", International Journal of Computer Application (0975-8887), Vol. 50-No.14, July 2012. 
Comparative Performance Analysis Of Mysql And Sqlite Relational Database Management Systems In Windows10 Environment

[5] J M Emison, "2014 State of Database Technology", Information Week, San Francisco, CA, Rep. R7770314, 2014.

[6] DB-Engines.com (2015, March 06). DB-Engines Ranking [Online]. Available: http://dbengines.com/en/ranking/relational+dbms.M. Young, The Technical Writer's Handbook. Mill Valley, CA: University Science, 1989.

[7] Lee J. (2013, November 30). Oracle vs. MySQL vs. SQL Server: A Comparison of Popular RDBMS [Online].

[8] Y. Bassil "A Comparative Study on the Performance of the Top DBMS Systems", Journal of Computer Science \& Research, Vol. 1-No. 1, February 2012.

[9] Chunyue Bi, "Research and Application of SQLite Embedded Database Technology", SCSIT, Zhejiang Wanli University, China.

[10] Michael Owens, The Definitive Guide to SQLite. USA: Apress, 2006, 341-362.

[11] SQLite homepage [EB/OL], http://www.sqlite.org.

[12] Wang Jingin, Wan Lixin, The comparison of Embedded Database Berkeley DB and SQLite, The application of SCM and Embedded System 2005, 28(2). 5-7. 